\title{
Association Between Epstein Barr Virus Infection and Lymphoproliferative Syndrome in a Renal Transplant Patient
}

\author{
Wissal Sahtout ${ }^{\mathrm{a}}$, Lilia Ben Lasfar ${ }^{\mathrm{a}, \mathrm{g}}$, Awatef Azzebi ${ }^{\mathrm{a}}$, Radhouane Mani ${ }^{\mathrm{b}}$, Nedia Arifac \\ Sawsan Kacem ${ }^{\text {d }}$, Ferdaous Sabria, Yosra Guedria ${ }^{\text {a }}$, Sanda Mrabet ${ }^{\mathrm{a}}$, Dorsaf Zellama ${ }^{\mathrm{a}}$, \\ Asma Fradia ${ }^{a}$, Salma Toumi ${ }^{\text {a }}$, Samira Ben Amor ${ }^{\mathrm{a}}$, Abderrahmen Khelife, \\ Moncef Moknif ${ }^{\text {, Halim Trabelsid }}{ }^{\text {, Abdellatif Achour }}{ }^{\mathrm{a}}$
}

\begin{abstract}
Epstein-Barr virus (EBV), a virus of the human Herpes viridae class, has a particular importance in renal transplantation because of its complex interaction with the immune system. Indeed, it causes both infectious mononucleosis (IM), immunopathological responses of the host, and at the extreme a malignant tumor, when the responses are altered. The lymphoproliferative syndromes induced by the EBV infection are lymphoid proliferations resulting from the loss of an EBV-specific cytotoxic T-cell response. About $10 \%$ of B-type lymphoproliferative syndromes including diffuse large Bcell lymphoma are EBV-positive. We report the case of a 23-yearold man who developed a tumor in the nasal cavity 6 months after renal transplantation. His nephropathy was membranoproliferative glomerulonephritis. The induction therapy indicated was antithymoglobulin (ATG) and solumedrol. In maintenance therapy, mycofenolate mofetil (MMF) and tacrolimus were used. Nasal biopsy has proved CD20-positive B-cell lymphoma. No other site of the disease has been identified. The immunosuppressive agents were modulated with a switch of tacrolimus by an m-TOR inhibitor and a progressive reduction of MMF. After three cycles of R CHOP followed by radiotherapy, he achieved complete remission with a stable graft function.
\end{abstract}

Keywords: Kidney transplantation; Viral infection; Epstein-Barr virus; Lymphoma; Immunosuppression

Manuscript submitted May 8, 2018, accepted June 4, 2018

aDepartment of Nephrology, Dialysis and Kidney Transplantation, Sahloul Hospital, Sousse, Tunisia

${ }^{\mathrm{b}}$ Department of Oto-Rhino-Laryngology, Sahloul Hospital, Sousse, Tunisia

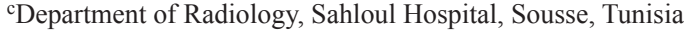

${ }^{\mathrm{d} D e p a r t m e n t}$ of Microbiology, Sahloul Hospital, Sousse, Tunisia

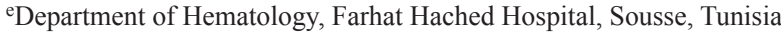

fDepartment of Anatomo-Pathology, Farhat Hached Hospital, Sousse, Tunisia

gCorresponding Author: Lilia Ben Lasfar, Department of Nephrology, Dialysis and Kidney Transplantation, Sahloul Hospital, Sousse, Tunisia.

Email: lililasfar@gmail.com

doi: https://doi.org/10.14740/wjnu352w

\section{Introduction}

The risk of malignant tumors is significantly increased in renal transplant patients. Neoplastic complications after renal transplantation are multiple, including particularly lymphoid malignancies, skin and lips carcinomas, vulva and perineum malignant tumors, cervical cancer, Kaposi's sarcoma, hepatocellular and renal cell carcinomas. A large proportion of these malignancies include post-transplantation lymphoproliferative disorders (SLP), which occur with a variable incidence of $1-8 \%$ in solid organ transplant recipients and $10 \%$ in kidney transplant recipients $[1,2]$.

They are recognized as an immunosuppression complication and are often associated with EBV, leading to uncontrolled proliferation of B lymphoid cells.

The majority of cases of SLP are non-Hodgkin's lymphomas (monomorphic SLP), and most of non-Hodgkin's lymphomas are large B-cell lymphomas [3]. We report the case of Epstein-Barr virus (EBV)-induced B-cell lymphoma after renal transplantation in remission.

\section{Case Report}

He is a 24-year-old kidney transplant patient on June 21, 2013 with a past medical history of congenital renal agenesis and left ureter stenosis. In 2005, renal failure and arterial hypertension were diagnosed. The renal histology showed a membranoproliferative glomerulonephritis. A preemptive kidney transplant was performed on June 21, 2013, from a living donor with six HLA mismatchs. He was EBV-negative and his donor was EBV-positive. Induction therapy included polyclonal antibodies and solumedrol and was followed by maintenance therapy (mycofenolate mofetil (MMF), tacrolimus and corticosteroid).

The evolution was favorable with stable renal function for nearly 5 months.

He maintained a serum creatinine level around $100-120$ $\mu \mathrm{mol} / \mathrm{L}$. At 2 months post-transplant, he was hospitalized for leukopenia induced by drug toxicity (MMF-Tacrolimus). On complete blood count, leucocytes were 2,430 elements $/ \mathrm{mm}^{3}$ 
and polymorphonuclear neutrophils were 1,440 element $/ \mathrm{mm}^{3}$. The residual blood level of tacrolimus was $13.2 \mathrm{ng} / \mathrm{mL}$ and MMF area under the curve was $27.44 \mathrm{mg} / \mathrm{h} / \mathrm{L}$.

Three months post-transplant, the viral serology tests for EBV were negative and positive for cytomegalovirus (CMV) (previous CMV infection). In November 2013, he was readmitted to the hospital for exploration of fever, headache and dehydration. A physical examination showed fever at $38.4{ }^{\circ} \mathrm{C}$, and the examination showed pain at frontal and maxillary sinus pressure without meningeal syndrome. The major abnormal findings were as follows: anemia $(9.9 \mathrm{~g} / \mathrm{dL})$, leucopenia $\left(2,250\right.$ cells $\left./ \mathrm{mm}^{3}\right)$, lymphopenia $\left(590\right.$ cells $\left./ \mathrm{mm}^{3}\right)$ and thrombocytopenia $\left(112,000\right.$ cells $\left./ \mathrm{mm}^{3}\right)$. Renal function had worsened at $180 \mu \mathrm{mol} / \mathrm{L}$, and glomerular filtration rate (GFR) was $51 \mathrm{~mL} / \mathrm{min} / 1.73 \mathrm{~m}^{2}$. Lactic acid dehydrogenase was $433 \mathrm{U} / \mathrm{L}$. And liver function was normal. For imaging, the abdominal ultrasound was without abnormalities.

The serologic tests for CMV and EBV were in favor of previous contact and lack of contact respectively.

Because CMV PCR was positive, the patient was putted on treatment: ganciclovir IV for 14 days relayed by valaciclovir PO. EBV PCR was positive. The evolution was unfavorable with persistence of fever, headache and arthralgia, leuconeutropenia and anemia. So, a bronchoalveolar lavage was performed and isolated, thereafter, a Mycobacterium tuberculosis, pseudomonas and Candida norvegensis.

The tuberculosis was systemic with multiple localizations: hematopoietic, ganglionic and hepatic, in addition to pulmonary localization. Indeed, the abdominal ultrasound showed a liver nodule $(2 \mathrm{~cm})$ and mesenteric adenomegaly. Liver biopsy showed granulomatous epithelioid and necrotic lesion. Antituberculous quadritherapy was started immediately based on ethambutol, rifampicin, izoniaside and pyazoline. In addition, an antibiotherapy based on (fortum, fosfomycin and cancidas) and an antifungal therapy (caspofungin) have been prescribed for bronchial infection.

During his hospitalization, the patient complained of right nasal obstruction; the imaging exploration revealed a thickening of nasopharynx posterior wall in both tomodensitometry and magnetic resonance imaging.

The patient thus had a nasal endoscopy showing a budding infected mass in the cavum. Histopathologically, the mass was a CD20-positive B-cell lymphoma.

A full body scan and an osteomedullary biopsy were normal and did not show any extension of the tumor.

Immediately, immunosuppression had been modulated with stopping of tacrolimus, switch to an inhibitor of m-TOR (sirolimus); the dose of mycophenolic acid was gradually lowered and thereafter stopped.

A bi-monthly chemotherapy with R-CHOP (rituximab, cyclophosphamide, doxorubicin, vincristine and prednisolone) was initiated followed by conventional radiotherapy with a total dose of 45 Gy divided into five sessions.

During chemotherapy, a control cavum scan had shown that the mass reduced to a discreet thickening of the posterolateral wall of the nasopharynx.

After a follow-up of 2 years, the patient remained in remission, with normal clinical, biological and imaging results. The creatinine level remained at $130 \mu \mathrm{mol} / \mathrm{L}$ and the proteinu- ria was $0.2 \mathrm{~g} / 24 \mathrm{~h}$.

On a morphological control, it was reported that the renal graft was increased in volume and at 6 months after the diagnosis of lymphoma, a biopsy of the graft was suggested but not performed because of disorders of hemostasis. Since that, the patient had no clinical or biological complications.

\section{Discussion}

SLP is a heterogeneous group of lymphoproliferation associated with immunosuppression (incidence is variable between $1 \%$ and $8 \%$ in the series of solid organ transplants and $0.3-3 \%$ in renal transplantation). Because of the heterogeneity of these SLP cases, there is no standardized classification. Nevertheless, there are currently two classes of poly- and mono-morphic SLP. Knowing that most mono-morphic cases are nonHodgkin's lymphomas including large cell B-cell lymphoma [2].

Most SLPs are related to EBV [2]. The virus was detected in $90 \%$ of injured cells during lymphoproliferation [2]. However, the group of EBV-induced SLP remains quite heterogeneous.

EBV is one of the most ubiquitous viruses; $90 \%$ of adults have previous contact and serological evidence of EBV infection in their lifetime [2]. This makes EBV an opportunistic pathogen that affects the immunocompromised patients.

In vitro, EBV infects non-active B cells and induces continuous and explosive proliferation $[2,3]$. It alters the expression of viral gene and escapes the immune system. In fact, EBV-infected cells are thought to avoid apoptosis by expressing EBV LMP-1 and LMP-2a [2]. In immunocompromised patients, in the case of absence of a specific cytotoxic action of EBV (T-cell response), the polyclonal expansion of infected lymphoblasts remains latent. Indeed, this process is caused by the administration of immunosuppression after transplantation [3].

According to Chen et al [4], 15 cases of SLP were retrospectively analyzed according to OMS classification (2008) of hematopoietic and lymphoid tissue tumors. Among 15 cases studied, 14 had hematopoietic stem cell transplantation and one had kidney transplantation. The average age was 30.4 years old. SLP were developed from 1.5 to 132 months after transplantation. Seven cases were poly-morphic SLP, with four cases containing a predominance of large cells. Four cases were mono-morphic SLP, of which three were diffuse large B-cell lymphomas, one was plasmablastic lymphoma, and one was mono-morphic and poly-morphic SLP. This study was conducted in China and reported that most of SLP are large B cells. The delay of disease onset was shorter in HIV-positive people [4].

Risk factors for the development of SLP include seronegativity of the recipient for EBV, use of intensive immunosuppressive agents, simultaneous CMV co-infection, young age and simultaneaous hepatitis $\mathrm{C}$ infection [2]. Of these risks, the most important is EBV seronegativity (the relative risk for SLP is 20 times higher for seronegatives). In our case, our patient has four risk factors: young age, seronegative EBV viral status 
before transplantation, immunosuppression and CMV infection.

As for its locatization, no case of large cell lymphoma B of the cavum has been listed in the literature. The other localizations of this histological type (B-cell lymphoma) analyzed in the literature were: central nervous system and skin.

For the neurological localization, the respective outcomes were different: a fatal outcome with a diagnostic confirmed on autopsy in the first case [5] and a total remission after use of rituximab, high doses of prednisolone and ganciclovir in the other case [6]. For cutaneous localization, B-cell lymphoma occurred after a long delay of renal transplantation (11 years), the treatment was based on rituximab and radiotherapy and the evolution of the lesion was slow; however, all cutaneous SLP must be imperatively treated [7].

B-cell lymphoma, after renal transplantation, can also be localized only in the graft. Indeed, Cobo et al have listed 16 cases of B-cell renal lymphoma without systemic disease. The most common clinical manifestations were graft dysfunction with fever. The purpose of this study of Cobo et al was to evaluate SLP exclusively localized in the transplanted kidney transplant. In the majority of patients included in this study, the diagnosis was made from allogenic transplant tissue [8].

In our case report, the lymphoma was localized in the cavum without invasive lesions and the evolution was favorable (complete remission) under chemo-radiotherapy. However, the hypertrophy of renal graft does not eliminate a secondary localization or a renal infiltrative form of the lymphoma. Nevertheless, the absence of graft dysfunction makes this hypothesis less valid.

Currently, treatment use chemo-radiotherapy of lymphoma, and other effective therapeutics: first sirolimus, then, reduction of the immunosuppression.

Indeed, sirolimus and its ester, water-soluble temsirolimus, are currently being evaluated in phases I and II clinical trials. These studies included solid tumors and hematopoietic malignancies. In a single-center study of 712 kidney transplants, a low dose of sirolimus (target blood level $5-8 \mathrm{ng} / \mathrm{mL}$ ) was shown to be sufficient to induce SLP regression, while maintaining renal graft function constant and preventing the acute rejection of allograft. According to recent trials, the conversion of calcineurin inhibitors to $\mathrm{m}$-TOR inhibitors is safe and also reduces the risk of chronic allograft nephropathy [9].

It is very difficult to determine whether SLP regression is related to the antineoplastic effect of sirolimus and/or due to decreased overall immunosuppression. A Mayo Clinic study showed that only $20-25 \%$ of patients with SLP achieved longterm remission after reduction or discontinuation of immunosuppression [10].

Multicentric studies are still needed to evaluate the real impact of m-TOR inhibitors on the proliferation of tumor cells.

The current goal is to detect early this neoplastic complication. An early diagnostic test has been proposed to detect "in time" the SLP by the detection of viral proteins called EBERS: polyadenylated RNA. EBV encodes two non-polyadenylated RNAs called EBV-encoded RNAs (Ebers). In a study conducted in 2015 [10], it was shown that EBV-positive SLP lesions are more likely to be developed in the early post-transplant period (the first year) with a relative risk (RR) of 1.36 and $\mathrm{P}$ $<0.001$.

Therefore, the SLP early onset is better prognosis. So the positive EBER test may not be bad information but rather imposes an earlier therapeutic alternative [10].

\section{Conclusions}

In this work, we report the case of a large cell lymphoma B, occurring at 1 year post-kidney transplant.

Risk factors for the development of SLP included seronegativity of the recipient, young age, use of potent immunosuppressive agents and simultaneous cytomegalovirus infection [11].

The originality of the case is the fact that the induced EBV lymphoma occurred in a seronegative recipient who received polyclonal antibodies in induction, the unique and unusual localization of this tumor and especially the favorable evolution under chemo-radiotherapy and rapamune with probable good graft tolerance despite the number of HLA mismatch and low immunosuppression.

Rapamune is an interesting anti-neoplastic and immunosuppressive agent that ensures regression of the tumor and prevention from both acute rejection and chronic allograft nephropathy.

\section{Conflict of Interest}

None.

\section{References}

1. Zadeh ZR, Makhdumi K, Lak SS. Epstein-Barr viral infection in renal allograft recipients: a single center experience. Saudi J Kidney Dis Transpl. 2006;17(3):351-354.

2. Choi JH, Ahn MJ, Oh YH, Han SW, Kim HJ, Lee YY, Kim IS. Epstein-Barr virus-associated Hodgkin's disease following renal transplantation. Korean J Intern Med. 2006;21(1):46-49.

3. Liu ID, Tan PL, Tan CX, Isa MS, Lau PY, Yeo WS, Chaturvedi S, et al. Metachronous B-cell and T-cell posttransplant lymphoproliferative disorders with features of chronic active Epstein-Barr virus infection. Am J Hematol. 2015;90(10):E204-205.

4. Chen DB, Wang Y, Song QJ, Shen DH. [Post-transplant lymphoproliferative disorder: a clinicopathologic study of 15 cases]. Zhonghua Bing Li Xue Za Zhi. 2012;41(9):607612.

5. Suzuki M, Kosugi I, Terada T, Shirakawa K, Suzuki H, Kono S, Miyajima H. A case of Epstein-Barr virus associated post-transplant lymphoproliferative disorder with CNS involvement: pathological findings at both biopsy and autopsy. Neuropathology. 2011;31(4):440-445.

6. Hansen PB, Al-Farra G. [Treatment of central nervous system lymphoma following transplantation using 
monoclonal antibody and ganciclovir]. Ugeskr Laeger. 2010;172(42):2907-2908.

7. Kudo K, Sonoda M, Sugimoto K, Koike M. [Cutaneous non-Hodgkin lymphoma of the leg occurring 11 years after renal transplantation]. Rinsho Ketsueki. 2009;50(2):107109.

8. Cobo F, Garcia C, Talavera P, Ruiz-Cabello F, Bravo J, Concha A. Diffuse large B-cell lymphoma in a renal allograft associated with Epstein-Barr virus in the recipient: a case report and a review of lymphomas presenting in a transplanted kidney. Clin Transplant. 2008;22(4):512519.

9. Boratynska M, Smolska D. Inhibition of mTOR by siroli- mus induces remission of post-transplant lymphoproliferative disorders. Transpl Int. 2008;21(6):605-608.

10. Khedmat H, Karbasi-Afshar R, Agah S, Ghamar-Chehreh ME, Amini M. A meta-analysis of potential relationship between Epstein-Barr-Encoded-RNA (EBER) and onset time of post-transplant lymphoproliferative disorders. Saudi J Kidney Dis Transpl. 2015;26(2):232-237.

11. Nose K, Oki T, Banno E, Sugimoto K, Nishioka T, Ochiai $\mathrm{K}$, Maekura S. The efficacy of EBER in situ hybridization (ISH) stain in PTLD (malignant diffuse large B-cell lymphoma) about 4 years after ABO-incompatible kidney transplantation: a case report. Int J Clin Exp Pathol. 2012;5(4):359-362. 